\title{
Assessment of Seven Clinical Tumor Markers in Diagnosis of Non-Small-Cell Lung Cancer
}

\author{
Zhong-qing Chen, Ling-sha Huang $(D$, and Bo Zhu $(\mathbb{D}$ \\ Department of Clinical Laboratory, Guangxi Medical University Affiliated Tumor Hospital, Nanning, \\ 530021 Guangxi Province, China \\ Correspondence should be addressed to Bo Zhu; bozhu@gxmu.edu.cn
}

Received 17 July 2018; Revised 12 August 2018; Accepted 9 September 2018; Published 11 December 2018

Academic Editor: Paola Gazzaniga

\begin{abstract}
Copyright ( 92018 Zhong-qing Chen et al. This is an open access article distributed under the Creative Commons Attribution License, which permits unrestricted use, distribution, and reproduction in any medium, provided the original work is properly cited.
\end{abstract}

\begin{abstract}
Background. The correlation between tumor markers (TM) and TNM stage of non-small-cell lung cancer (NSCLC) has not been widely reported. Methods. TM levels (CEA, CA125, CA15-3, CA19-9, CA72-4, CYFRA21-1, and SCC-Ag) in 424 cases of lung adenocarcinoma (LAC), 166 cases of lung squamous cell carcinoma (LSCC), and 103 cases of benign chest disease (BCD) were analyzed before treatment. Results. By Kendall's tau-b correlation analysis, CEA, CA125, CA15-3, CA19-9, CA72-4, CYFRA21-1, and SCC-Ag were correlated with T stage of LAC $(r=0.235, p<0.05 ; r=0.298, p<0.05 ; r=0.254, p<0.05 ; r=0.063, p<0.05$; $r=0.080, p<0.05 ; r=0.268, p<0.05$; and $r=0.080, p<0.05)$. CEA, CA125, CA15-3, CA19-9, CA72-4, and CYFRA21-1 were correlated with N stage of LAC $(r=0.356, p<0.05 ; r=0.415, p<0.05 ; r=0.340, p<0.05 ; r=0.117, p<0.05 ; r=0.175, p<0.05$; and $r=0.351, p<0.05)$. CEA, CA125, CA15-3, CA19-9, CA72-4, and CYFRA21-1 were correlated with M stage of LAC $(r=0.365, p<0.05 ; r=0.353, p<0.05 ; r=0.293, p<0.05 ; r=0.135, p<0.05 ; r=0.169, p<0.05 ;$ and $r=0.312, p<0.05)$. CA125, CYFRA21-1, and SCC-Ag were correlated with T stage of LSCC $(r=0.202, p<0.05 ; r=0.233, p<0.05 ;$ and $r=0.099$, $p<0.05)$. CA125 and CYFRA21-1 were correlated with N stage of LSCC $(r=0.178, p<0.05$ and $r=0.284, p<0.05)$. CA125, CA15-3, and CYFRA21-1 were correlated with M stage of LSCC $(r=0.214, p<0.05 ; r=0.152, p<0.05$; and $r=0.213, p<0.05)$. Combining hazard ratio, AUC, sensitivity, specificity, NPV, and PPV, it was concluded that CEA and CYFRA21-1were the most related TM of LAC. SCC-Ag and CYFRA21-1 were the most related TM of LSCC. Conclusions. CEA combined with CYFRA211 contributed to auxiliary diagnosis of LAC. CYFRA21-1 combined with SCC-Ag contributed to auxiliary diagnosis of LSCC. CEA, CA125, CA15-3, CA19-9, CA72-4, and CYFRA21-1 were correlated with primary tissue and metastasis of LAC. CA125 and CYFRA21-1 were correlated with primary tissue and metastasis of LSCC.
\end{abstract}

\section{Introduction}

Data released by the National Cancer Registry (NCCR) of China in 2018-2015 showed that primary lung cancer was the first cancer in morbidity and mortality for four consecutive years [1-3]. The diagnosis of early-stage non-small-cell lung cancer (NSCLC) was the first step toward successful clinical therapy and increased patient survival. Making full use of the existing means to diagnose early NSCLC has been concerned. Histopathological examination is the gold standard for the diagnosis of NSCLC. However, due to the invasiveness of histopathological examination, chest computed tomography and tumor markers (TM) test preceded histopathological examination. The TM test results were increasingly influencing decisions on tumor screening, initial treatment, and follow-up for NSCLC based on the advantages of minimal trauma, good repeatability, simplicity, and rapidity. TM can be used to assist diagnosis and differential diagnosis and to understand the possible pathological types of NSCLC. In the clinical diagnosis of NSCLC, carcinoembryonic antigen (CEA), cytokeratin 19 fragments antigen (CYFRA21-1), and squamous cell carcinoma antigen (SCC$\mathrm{Ag}$ ) have been used as reference TM for NSCLC. Other common TM also increased in NSCLC, including carbohydrate antigen 125 (CA125), CA15-3, CA19-9, and CA72-4. Previous studies have shown that TM level was correlated with 
pathological types, primary tissues [4], lymph node metastasis $[5,6]$, and distant metastasis [7]. Combination of TM can improve the diagnostic accuracy of NSCLC [8-10]. However, the points of view on combination of TM were not consistent. The correlation between TM and TNM stage of NSCLC has not been widely reported. Our research came from daily clinical practice. The TM levels came from before treatment since TM vary significantly before and after treatment $[11,12]$. We chose benign chest diseases (BCD) that were easily confused with NSCLC as a control. We systematically discussed the characteristics of seven TM in pathological type, TNM stage, and diagnosis of NSCLC.

\section{Materials and Methods}

2.1. Patients. This study was approved by the ethics committee of the Guangxi Medical University Affiliated Tumor Hospital with an ethics approval number of LW2018011. When the patients suspected they had lung cancer, they were first admitted to the thoracic tumor surgery department. We systematically reviewed all patients who had been hospitalized in the thoracic tumor surgery department from October 2014 to August 2017. Cases with other malignancies and/or no pathology and/or no image were excluded. NSCLC and BCD were all first diagnosed. The diagnosis of NSCLC was established by histopathology. NSCLC was staged according to the 2009 seventh edition of the tumor-nodes-metastasis (TNM) classification for lung cancer [13-15]. The diagnosis of BCD was established by histopathology or pathogeny. BCD cases were not treated with any therapy. NSCLC cases had not been treated with any antitumor therapy, such as surgery, chemotherapy, radiotherapy, biological therapy, endocrine therapy, Chinese medicine treatment, hyperthermia, and radiofrequency ablation therapy. Complete and detailed case information were available for all cases. A total of 424 cases of lung adenocarcinoma (LAC), 166 cases of lung squamous cell carcinoma (LSCC), and 103 BCD patients were included in this study (Table 1).

2.2. Laboratory Assays. Each subject agreed to undergo fasting blood tests the next morning after admission, which was routine treatment. The test was completed on the day of blood drawing to avoid attenuation. All assays were performed according to instrument and reagent specifications. CEA, CA125, CA15-3, CA19-9, CA72-4, CYFRA21-1, and SCC-Ag were measured using ARCHITECTi2000 ${ }_{S R}$ automatic electrochemical luminescence instrument and supporting reagents (Abbott Laboratories, Chicago). TM level above the following values was considered abnormal: CEA, $5 \mathrm{ng} / \mathrm{ml}$; CA125, $35 \mathrm{ng} / \mathrm{ml}$; CA15-3, $30 \mathrm{ng} / \mathrm{ml}$; CA19-9, $37 \mathrm{ng} / \mathrm{ml}$; CA72-4, $5.3 \mathrm{ng} / \mathrm{ml}$; CYFRA 21-1, $3.3 \mathrm{ng} / \mathrm{ml}$; and SCC-Ag, $1.5 \mathrm{ng} / \mathrm{ml}$.

2.3. Statistical Analysis. IBM SPSS21.0 and MedCalc 18.2 software were applied for statistical analysis. The levels adopted for significance were $p<0.05$ (two tailed). The calculation of sample content: $n=\mu_{\mathrm{a}}{ }^{2} \mathrm{p}(1-\mathrm{p}) / \delta^{2}$, the sample size of the case group and the control group were all 96 , and the sample size of the study met the requirements. The normality
TABLE 1: Characteristics of the study subjects.

\begin{tabular}{|c|c|c|c|}
\hline Parameters & $\begin{array}{l}\text { NSCLC } \\
n=590\end{array}$ & Parameters & $\begin{array}{c}\text { BCD } \\
n=103\end{array}$ \\
\hline Gender & & Gender & \\
\hline Male & 402 & Male & 55 \\
\hline Female & 188 & Female & 48 \\
\hline Age (year) & & Age (year) & \\
\hline Range & $28-86$ & Range & $18-76$ \\
\hline Mean & 57.7 & Mean & 53.9 \\
\hline Pathological type & & Disease & \\
\hline LAC & 424 & Hemangioma & 4 \\
\hline LSCC & 166 & Hamartoma & 6 \\
\hline T stage & & $\begin{array}{l}\text { Inflammatory } \\
\text { pseudotumor }\end{array}$ & 8 \\
\hline $\mathrm{T} 1$ & 119 & Fibrous nodules & 6 \\
\hline $\mathrm{T} 2$ & 260 & Tuberculosis & 22 \\
\hline $\mathrm{T} 3$ & 176 & Infection & 33 \\
\hline $\mathrm{T} 4$ & 35 & Lymphoproliferation & 2 \\
\hline $\mathrm{N}$ stage & & Cysts & 12 \\
\hline No & 172 & Thymoma & 3 \\
\hline N1 & 65 & Substernal thyroid & 1 \\
\hline $\mathrm{N} 2$ & 222 & Amyloidosis & 1 \\
\hline N3 & 131 & Lipoma & 1 \\
\hline M stage & & Schwannoma & 4 \\
\hline M0 & 376 & - & - \\
\hline M1 & 214 & - & - \\
\hline
\end{tabular}

BCD: benign chest disease; NSCLC: non-small-cell lung cancer; LAC: lung adenocarcinoma; LSCC: lung squamous cell carcinoma.

test used skewness coefficient $(s k)$ and kurtosis coefficient (ku). The TM data was not normal distribution. Mann-Whitney $U$ test of nonparametric rank sum test was used to compare the median of two groups. Chi-square test $\left(\chi^{2}\right)$ was used to compare the positive rates of two groups. The correlation between TM level and TNM stage of NSCLC was analyzed by Kendall's tau-b correlation analysis. The levels of TM may be influenced by $\mathrm{T}$ stage, $\mathrm{N}$ stage, $\mathrm{M}$ stage, and other factors, so the correlation coefficient was low. In this paper, the correlation analysis was based on $p<0.05$ not the correlation coefficient. With BCD as a control, binary logistic regression was used to calculate the hazard ratios of TM in NSCLC. Applying the receiver operating characteristic curve (ROC curve) to calculate the area under the curve (AUC) of TM in NSCLC, $z$ test was used to compare AUC. Sensitivity, specificity, negative predictive value (NPV), and positive predictive value (PPV) were calculated.

\section{Results}

3.1. Patients' Characteristics. A total of 590 NSCLC patients and 103 BCD patients were included in the study. Table 1 summarizes patients' characteristics. Of note, the proportion of advanced NSCLC was higher than that of early NSCLC at initial diagnosis (Table 1). 
TABLE 2: The median and positive rates of tumor markers in benign chest disease (BCD), lung adenocarcinoma (LAC), and lung squamous cell carcinoma (LSCC).

\begin{tabular}{lcccccc}
\hline Parameters & BCD & LAC & LSCC & $p 1$ & $p 2$ & $p 3$ \\
& $n=103$ & $n=424$ & $n=166$ & value & value & value \\
\hline Median (ng/ml) & & & & & & \\
CEA & 2.1 & 7.4 & 3.3 & 0.000 & 0.000 & 0.000 \\
CA125 & 12.8 & 22.5 & 20.0 & 0.000 & 0.000 & 0.074 \\
CA15-3 & 12.3 & 20.4 & 15.0 & 0.000 & 0.035 & 0.000 \\
CA19-9 & 10.5 & 14.0 & 10.6 & 0.010 & 0.397 & 0.000 \\
CA72-4 & 1.3 & 2.0 & 1.8 & 0.000 & 0.019 & 0.164 \\
CYFRA21-1 & 2.3 & 4.1 & 7.6 & 0.000 & 0.000 & 0.000 \\
SCC-Ag & 0.7 & 0.6 & 1.3 & 0.443 & 0.000 & 0.000 \\
Positive rate (\%) & & & & & & \\
CEA & 7.8 & 61.8 & 23.5 & 0.000 & 0.001 & 0.000 \\
CA125 & 8.7 & 40.6 & 28.9 & 0.000 & 0.000 & 0.009 \\
CA15-3 & 2.9 & 30.2 & 9.0 & 0.000 & 0.051 & 0.000 \\
CA19-9 & 5.8 & 19.8 & 5.4 & 0.001 & 0.888 & 0.000 \\
CA72-4 & 11.7 & 21.7 & 16.9 & 0.022 & 0.242 & 0.190 \\
CYFRA21-1 & 13.6 & 61.6 & 88.6 & 0.000 & 0.000 & 0.000 \\
SCC-Ag & 3.9 & 8.5 & 39.8 & 0.113 & 0.000 & 0.000 \\
\hline
\end{tabular}

$p 1$ value: $B C D$ vs $L A C, p 2$ value: BCD vs LSCC, $p 3$ value: $L A C$ vs LSCC.

3.2. Distribution Characteristics of Tumor Markers in NonSmall-Cell Lung Cancer and Benign Chest Disease. The positive rates of TM in BCD were CYFRA21-1 > CA72-4> CA125 $>$ CEA $>$ CA19-9 $>$ SCC-Ag $>$ CA15-3. The median of TM in BCD was within normal range. The median and positive rates of CEA, CA125, CA15-3, CA19-9, CA72-4, and CYFRA21-1 in LAC were higher than those in BCD $(p<0.05)$. The median and positive rates of CEA, CA125, CYFRA21-1, and SCC-Ag in LSCC were higher than those in $\operatorname{BCD}(p<0.05)$. Among them, the median and positive rates of CEA, CA125, and CYFRA21-1 in both LAC and LSCC were higher than those in $\mathrm{BCD}(p<0.05)$. The median and positive rates of CEA, CA15-3, and CA19-9 in LAC were higher than those in LSCC $(p<0.05)$. The median and positive rates of CYFRA21-1 and SCC-Ag in LSCC were higher than those in LAC $(p<0.05)$ (Table 2).

3.3. Correlation Analysis of Tumor Markers and TNM Stage in Lung Adenocarcinoma and in Lung Squamous Cell Carcinoma. Through Kendall's tau-b correlation analysis, it was found that CEA, CA125, CA15-3, CA19-9, CA72-4, CYFRA21-1, and SCC-Ag were correlated with T stage of LAC $(r=0.235, p<0.05 ; r=0.298, p<0.05 ; r=$ $0.254, p<0.05 ; r=0.063, p<0.05 ; r=0.080, p<0.05 ; r=$ $0.268, p<0.05$; and $r=0.080, p<0.05)$. CEA, CA125, CA15-3, CA19-9, CA72-4, and CYFRA21-1 were correlated with N stage of LAC $(r=0.356, p<0.05 ; r=0.415, p<0.05$; $r=0.340, p<0.05 ; r=0.117, p<0.05 ; r=0.175, p<0.05$; and $r=0.351, p<0.05)$. CEA, CA125, CA15-3, CA19-9, CA72-4, and CYFRA21-1 were correlated with $M$ stage of LAC $(r=0.365, p<0.05 ; r=0.353, p<0.05 ; r=0.293$,
TABLE 3: Correlation analysis of tumor markers and TNM stage in lung adenocarcinoma.

\begin{tabular}{lcccccc}
\hline \multirow{2}{*}{ Parameters } & \multicolumn{2}{c}{ T stage } & \multicolumn{2}{c}{ N stage } & \multicolumn{2}{c}{ M stage } \\
& $r$ & $p$ value & $r$ & $p$ value & $r$ & $p$ value \\
\hline CEA & 0.235 & 0.000 & 0.356 & 0.000 & 0.365 & 0.000 \\
CA125 & 0.298 & 0.000 & 0.415 & 0.000 & 0.353 & 0.000 \\
CA15-3 & 0.254 & 0.000 & 0.340 & 0.000 & 0.293 & 0.000 \\
CA19-9 & 0.063 & 0.045 & 0.117 & 0.001 & 0.135 & 0.000 \\
CA72-4 & 0.080 & 0.015 & 0.175 & 0.000 & 0.169 & 0.000 \\
CYFRA21-1 & 0.268 & 0.000 & 0.351 & 0.000 & 0.312 & 0.000 \\
SCC-Ag & 0.080 & 0.016 & 0.000 & 0.497 & -0.028 & 0.245 \\
\hline
\end{tabular}

TABLE 4: Correlation analysis of tumor markers and TNM stage in lung squamous cell carcinoma.

\begin{tabular}{lcccccc}
\hline \multirow{2}{*}{ Parameters } & \multicolumn{2}{c}{ T stage } & \multicolumn{2}{c}{$\mathrm{N}$ stage } & \multicolumn{2}{c}{ M stage } \\
& $r$ & $p$ value & $\mathrm{r}$ & $p$ value & $r$ & $p$ value \\
\hline CEA & 0.078 & 0.092 & 0.038 & 0.263 & 0.020 & 0.379 \\
CA125 & 0.202 & 0.000 & 0.178 & 0.001 & 0.214 & 0.000 \\
CA15-3 & -0.045 & 0.222 & 0.013 & 0.416 & 0.152 & 0.008 \\
CA19-9 & 0.044 & 0.227 & 0.029 & 0.312 & 0.047 & 0.230 \\
CA72-4 & 0.061 & 0.152 & 0.051 & 0.193 & -0.013 & 0.419 \\
CYFRA21-1 & 0.233 & 0.000 & 0.284 & 0.000 & 0.213 & 0.000 \\
SCC-Ag & 0.099 & 0.046 & 0.046 & 0.217 & 0.035 & 0.290 \\
\hline
\end{tabular}

$p<0.05 ; r=0.135, p<0.05 ; r=0.169, p<0.05 ;$ and $r=$ $0.312, p<0.05)$ (Table 3).

Through Kendall's tau- $b$ correlation analysis, it was found that CA125, CYFRA21-1, and SCC-Ag were correlated with T stage of LSCC $(r=0.202, p<0.05 ; r=0.233, p<0.05$; and $r=0.099, p<0.05)$. CA125 and CYFRA21-1 were correlated with N stage of LSCC $(r=0.178, p<0.05$ and $r=0.284$, $p<0.05)$. CA125, CA15-3, and CYFRA21-1 were correlated with M stage of LSCC $(r=0.214, p<0.05 ; r=0.152, p<$ 0.05 ; and $r=0.213, p<0.05$ ) (Table 4).

3.4. Diagnostic Characteristics of Tumor Markers in NonSmall-Cell Lung Cancer. The AUC of CEA, CA12-5, CA153, CA72-4, and CYFRA21-1 was statistically significant in LAC (all $p<0.05$ ). The AUC of CEA in LAC was significantly higher than CA125 $(z=5.361, p<0.05)$, CA15-3 $(z=4.629, p<0.05)$, CA72-4 $(z=6.598, p<0.05)$, and CYFRA21-1 $(z=2.162, p<0.05)$. The AUC of CYFRA211 in LAC was significantly higher than CA12-5 $(z=3.147$, $p<0.05)$, CA15-3 $(z=2.243, p<0.05)$, and CA72-4 $(z=$ $4.641, p<0.05)$. The AUC of CEA, CA125, CYFRA21-1, and SCC-Ag was statistically significant in LSCC (all $p<$ $0.05)$. The AUC of CYFRA21-1 in LSCC was significantly higher than CEA $(z=6.463, p<0.05)$, CA125 $(z=7.112$, $p<0.05)$, and SCC-Ag $(z=4.993, p<0.05)$. The AUC of SCC-Ag in LSCC was significantly higher than CEA $(z=$ $2.093, p<0.05)$ and CA125 $(z=2.288, p<0.05)$. Combining hazard ratio, AUC, sensitivity, specificity, NPV, and PPV, it 
was concluded that CEA and CYFRA21-1were the most related TM of LAC and SCC-Ag and CYFRA21-1 were the most related TM of LSCC (Table 5, Figure 1).

\section{Discussions}

TM was also expressed in BCD. Increased TM in BCD may be correlated with inflammation and disease severity [16]. Seven TM in BCD are out of the normal range to varying degrees. In this study, the parallel positive rate of seven $\mathrm{TM}$ in $\mathrm{BCD}$ was $41.7 \%$ and the tandem positive rate was $55.3 \%$. The parallel positive rate of seven TM in LAC was $86.5 \%$ and the tandem positive rate was $245.3 \%$. The parallel positive rate of seven TM in LSCC was $93.4 \%$ and the tandem positive rate was $212.1 \%$. It was shown that TM in BCD mainly increased by one, and that in NSCLC mainly increased by two or more. The median of seven TM in BCD was within the normal range. The median of CEA and CYFRA21-1 in LAC and the median of CYFRA21-1 and SCC-Ag in LSCC had approached or exceeded the normal range. The median and positive rates of CEA, CA125, CA15-3, CA19-9, and CYFRA21-1 in LAC were higher than those in BCD $(p<0.05)$. The median and positive rates of CEA, CA125, CYFRA21-1, and SCC-Ag in LSCC were higher than those in $\mathrm{BCD}(p<0.05)$. It was shown that TM was lowly expressed in BCD and highly expressed in NSCLC.

The expression of CEA in primary tissue and lymph node metastasis of LAC was higher than that in other NSCLC types [17]. CEA was highly expressed in nonlepidic dominant histologic subtype [18], adenocarcinoma tissues coexisting with bullae or honeycomb cysts [19] and welldifferentiated adenocarcinoma tissues [20]. The expression of CEA was also correlated with TNM stage of NSCLC [21, 22]. CEA was correlated with $\mathrm{T}$ stage, $\mathrm{N}$ stage, and $\mathrm{M}$ stage of LAC $(r=0.235, p<0.05, r=0.356, p<0.05$, and $r=$ $0.365, p<0.05)$. It was shown that the level of CEA was correlated with primary tissue, lymphatic metastasis, and distant metastasis of LAC. However, CEA was not correlated with TNM stage of LSCC $(p>0.05)$. The correlation between CA19-9, CA72-4, and TNM stage in LAC and LSCC were similar to that of CEA; the correlation coefficients were lower. CA19-9 increased in patients with infectious lung disease [23]. NSCLC, especially advanced NSCLC, was often accompanied by severe infection. Therefore, CA19-9 can help identify NSCLC stage. In addition, CA19-9 was highly expressed in certain pathological types of LAC [24-26]. CA72-4 had not been reported in NSCLC.

CYFRA21-1 expressed in respiratory epithelial cells and has been detected in NSCLC tissue [27]. The increase of CYFRA21-1 in blood was more common in NSCLC, and its sensitivity was highest in LSCC and was considered as the best TM for LSCC $[27,28]$. CYFRA21-1 was an independent predictor of NSCLC metastasis [7]. In this study, CYFRA211 was correlated with $\mathrm{T}$ stage, $\mathrm{N}$ stage, and $\mathrm{M}$ stage of both LAC and LSCC $(r=0.268, p<0.05 ; r=0.351, p<0.05 ; r=$ $0.312, p<0.05 ; r=0.233, p<0.05 ; r=0.284, p<0.05$; and $r=0.231, p<0.05)$. CYFRA21-1 was risk index for both LAC and LSCC $(p<0.05)$. In LAC, the AUC of CYFRA21-1
TABLE 5: The diagnostic characteristics of tumor markers in lung adenocarcinoma (LAC) and lung squamous cell carcinoma (LSCC).

\begin{tabular}{lcccccc}
\hline Parameters & $\begin{array}{c}\text { Hazard } \\
\text { ratio }\end{array}$ & AUC & Sensitivity & Specificity & NPV & PPV \\
\hline LAC & & & & & & \\
$(n=424)$ & & & & & & \\
CEA & $1.34^{*}$ & $0.85^{*}$ & 61.8 & 92.2 & 37.0 & 97.0 \\
CA125 & 1.00 & $0.69^{*}$ & 40.6 & 91.3 & 27.2 & 95.0 \\
CA15-3 & 1.03 & $0.72^{*}$ & 30.2 & 97.1 & 25.3 & 97.7 \\
CA19-9 & 1.00 & 0.58 & 19.8 & 94.2 & 22.2 & 93.3 \\
CA 72-4 & 1.01 & $0.61^{*}$ & 21.7 & 88.3 & 21.5 & 88.5 \\
CYFRA21-1 & $1.43^{*}$ & $0.79^{*}$ & 61.6 & 86.4 & 35.3 & 94.9 \\
SCC-Ag & 1.03 & 0.48 & 8.5 & 96.1 & 20.3 & 90.0 \\
LSCC & & & & & & \\
$(n=166)$ & & & & & & \\
CEA & 1.09 & $0.70^{*}$ & 23.5 & 92.2 & 42.8 & 83.0 \\
CA125 & 1.00 & $0.68^{*}$ & 28.9 & 91.3 & 44.3 & 84.2 \\
CA15-3 & 1.03 & 0.58 & 9.0 & 97.1 & 39.8 & 83.3 \\
CA19-9 & 1.00 & 0.47 & 5.4 & 94.2 & 38.2 & 60.0 \\
CA72-4 & 1.03 & 0.59 & 16.9 & 88.3 & 39.7 & 70.0 \\
CYFRA21-1 & $2.26^{*}$ & $0.93^{*}$ & 88.6 & 86.4 & 82.4 & 91.3 \\
SCC-Ag & $2.28^{*}$ & $0.78^{*}$ & 39.8 & 96.1 & 49.7 & 94.3 \\
\hline
\end{tabular}

${ }^{*} p$ value $<0.05$.

(0.79) was second only to CEA (0.85). In LSCC, the AUC of CYFRA21-1 was the largest (0.93). Therefore, CYFRA21-1 was a more reliable TM for the diagnosis of NSCLC. The level of CYFRA21-1 was correlated with primary tissue, lymphatic metastasis, and distant metastasis of NSCLC. The expression of CA125 was not related to the degree of malignancy [29] but related to the degree of tissue differentiation. CA125 was highly expressed in lessdifferentiated tumor [20]. CA125 was positively correlated with postoperative metastasis [5] and postoperative recurrence in NSCLC [30]. Similar to CYFRA21-1, CA125 was correlated with $\mathrm{T}$ stage, $\mathrm{N}$ stage, and $\mathrm{M}$ stage of both LAC and LSCC $(r=0.298, p<0.05 ; r=0.415, p<0.05$; $r=0.353, p<0.05 ; r=0.298, p<0.05 ; r=0.415, p<0.05$; and $r=0.353, p<0.05)$. The expression CA125 in NSCLC was also correlated with primary tissue, lymphatic metastasis, and distant metastasis.

The expression of SCC-Ag was correlated with differentiation degree of squamous cell carcinoma tissue. SCC-Ag was highly expressed in well-differentiated squamous cell carcinoma tissues and lowly expressed in poorly differentiated squamous cell carcinoma tissues [31]. The level of SCC-Ag was correlated with $\mathrm{T}$ stage of both LAC and LSCC $(r=0.080, p<0.05 ; r=0.099, p<0.05)$. The level of SCCAg may be correlated with the highly differentiated squamous cell carcinoma of the lung. Due to the low sensitivity, SCC-Ag provided no additional value when used in combination with CYFRA21-1 to diagnose NSCLC [32]. Compared 

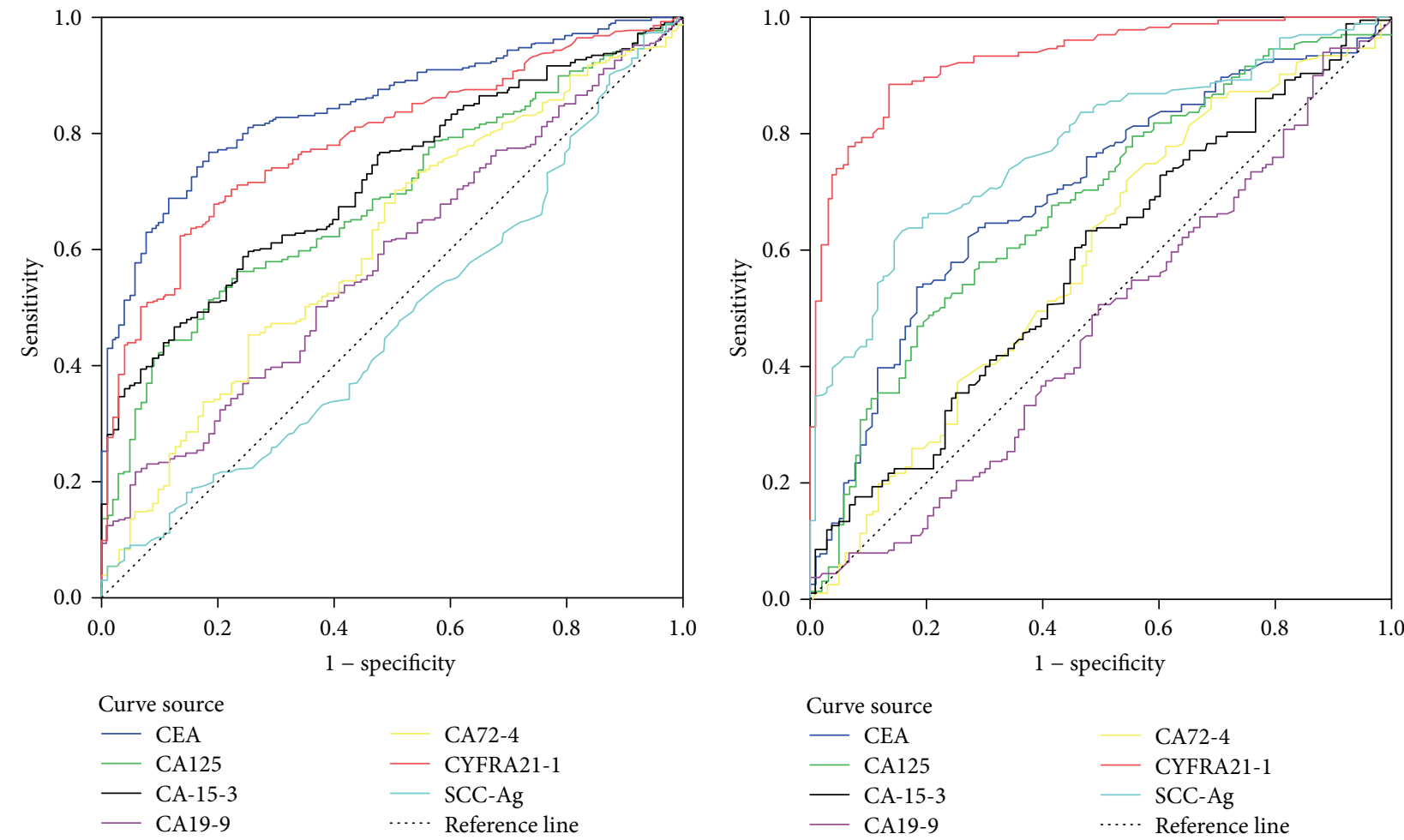

(a)

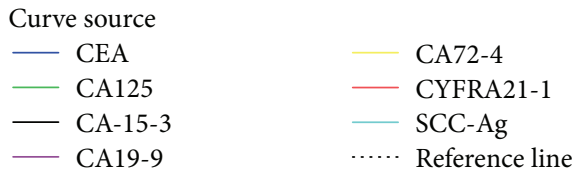

(b)

FIGURE 1: (a) ROC curve of tumor markers in lung adenocarcinoma. (b) ROC curve of tumor markers in lung squamous cell carcinoma.

with CYFRA21-1, SCC-Ag has lower sensitivity (39.8\%) but higher specificity (96.1\%) for LSCC. SCC-Ag was still a widely used TM to identify pathological types of NSCLC and still an effective method for the identification of benign and malignant solitary pulmonary nodules [33].

CA15-3 was a soluble form of mucin-1 serum shedding [34]. Mucin-1 expression may be associated with nonsquamous cell carcinoma tissue [35]. CA15-3 level was correlated with $\mathrm{T}$ stage, $\mathrm{N}$ stage, and $\mathrm{M}$ stage of LAC $(r=0.254, p<0.05 ; r=0.340, p<0.05$; and $r=0.293$ , $p<0.05)$. CA15-3 was also correlated with $\mathrm{M}$ stage of LSCC $(r=0.168, p<0.05)$. CA15-3 in LAC may be correlated with primary tissue, lymph node metastasis, and distant metastasis. CA15-3 in LSCC may indicate distant metastasis. However, Liu et al. [36] reported that mucin-1 mRNA in NSCLC peripheral blood cannot be used as a reliable TM of metastasis. The value of CA15-3 in diagnosis of NSCLC still needs further study.

\section{Conclusion}

CEA combined with CYFRA21-1 contributed to auxiliary diagnosis of LAC. CYFRA21-1 combined with SCC-Ag contributed to auxiliary diagnosis of LSCC. CEA, CA125, CA15-3, CA19-9, CA72-4, and CYFRA21-1 were correlated with primary tissue and metastasis of LAC. CA125 and CYFRA21-1 were correlated with primary tissue and metastasis of LSCC.

\section{Data Availability}

The data availability statement is listed in the manuscript.

\section{Ethical Approval}

This study was approved by the ethics committee of the Guangxi Medical University Affiliated Cancer Hospital with an ethics approval number of LW2018011.

\section{Conflicts of Interest}

The authors have no conflicts of interest to declare.

\section{Authors' Contributions}

Zhong-qing Chen and Ling-sha Huang contributed equally to this work.

\section{Acknowledgments}

This work was supported by the National High Technology Research and Development Program of China (863 Program) (grant number 2014AA022304 to Bo Zhu).

\section{Supplementary Materials}

Supplemental material is raw data. The non-small-cell lung cancer sheet includes gender, age, pathological type ( $1=$ lung adenocarcinoma and $2=$ lung squamous cell carcinoma), 
tumor marker levels (CEA, CA125, CA15-3, CA19-9, CA72-4, CYFRA211, and SCC-Ag), and TNM stage. The benign chest disease sheet includes gender, age, tumor marker levels (CEA, CA125, CA15-3, CA19-9, CA72-4, CYFRA211, and SCC-Ag), and diagnostic classification. (Supplementary Materials)

\section{References}

[1] R. Zheng, H. Zeng, S. Zhang, and W. Chen, "Estimates of cancer incidence and mortality in China, 2013," Chinese Journal of Cancer, vol. 36, no. 1, p. 66, 2017.

[2] W. Chen, R. Zheng, H. Zeng, and S. Zhang, "The incidence and mortality of major cancers in China, 2012," Chinese Journal of Cancer, vol. 35, no. 1, p. 73, 2016.

[3] W. Chen, R. Zheng, H. Zeng, S. Zhang, and J. He, "Annual report on status of cancer in China, 2011," Chinese Journal of Cancer Research, vol. 27, no. 1, pp. 2-12, 2015.

[4] Y. Xu, H. Sun, Z. Zhang, A. Song, W. Wang, and X. Lu, "Assessment of relationship between CT features and serum tumor marker index in early-stage lung adenocarcinoma," Academic Radiology, vol. 23, no. 11, pp. 1342-1348, 2016.

[5] M. Pollán, G. Varela, A. Torres et al., "Clinical value of p53, c-erbB-2, CEA and CA125 regarding relapse, metastasis and death in resectable non-small cell lung cancer," International Journal of Cancer, vol. 107, no. 5, pp. 781790, 2003.

[6] A. Bugalho, C. Martins, S. S. Dias et al., "Cytokeratin 19, carcinoembryonic antigen, and epithelial cell adhesion molecule detect lung cancer lymph node metastasis in endobronchial ultrasound-guided transbronchial aspiration samples," Clinical Lung Cancer, vol. 14, no. 6, pp. 704-712, 2013.

[7] J. L. Cabrera-Alarcon, A. Carrillo-Vico, J. D. Santotoribio et al., "CYFRA 21-1 as a tool for distant metastasis detection in lung cancer," Clinical Laboratory, vol. 57, no. 11-12, pp. 1011-1014, 2011.

[8] R. Molina, R. M. Marrades, J. M. Augé et al., "Assessment of a combined panel of six serum tumor markers for lung cancer," American Journal of Respiratory and Critical Care Medicine, vol. 193, no. 4, pp. 427-437, 2016.

[9] R. Molina, X. Bosch, J. M. Auge et al., "Utility of serum tumor markers as an aid in the differential diagnosis of patients with clinical suspicion of cancer and in patients with cancer of unknown primary site," Tumor Biology, vol. 33, no. 2, pp. 463-474, 2012.

[10] K. Okamura, K. Takayama, M. Izumi, T. Harada, K. Furuyama, and Y. Nakanishi, "Diagnostic value of CEA and CYFRA 21-1 tumor markers in primary lung cancer," Lung Cancer, vol. 80, no. 1, pp. 45-49, 2013.

[11] S. Holdenrieder, J. von Pawel, E. Dankelmann et al., "Nucleosomes, ProGRP, NSE, CYFRA 21-1, and CEA in monitoring first-line chemotherapy of small cell lung cancer," Clinical Cancer Research, vol. 14, no. 23, pp. 7813-7821, 2008.

[12] S. Holdenrieder, B. Wehnl, K. Hettwer, K. Simon, S. Uhlig, and F. Dayyani, "Carcinoembryonic antigen and cytokeratin19 fragments for assessment of therapy response in nonsmall cell lung cancer: a systematic review and meta-analysis," British Journal of Cancer, vol. 116, no. 8, pp. 1037-1045, 2017.

[13] P. Goldstraw, J. Crowley, K. Chansky et al., "The IASLC lung cancer staging project: proposals for the revision of the TNM stage groupings in the forthcoming (seventh) edition of the
TNM classification of malignant tumours," Journal of Thoracic Oncology, vol. 2, no. 8, pp. 706-714, 2007.

[14] P. A. Groome, V. Bolejack, J. J. Crowley et al., “The IASLC lung cancer staging project: validation of the proposals for revision of the $\mathrm{T}, \mathrm{N}$, and $\mathrm{M}$ descriptors and consequent stage groupings in the forthcoming (seventh) edition of the TNM classification of malignant tumours," Journal of Thoracic Oncology, vol. 2, no. 8, pp. 694-705, 2007.

[15] W. D. Travis, D. J. Giroux, K. Chansky et al., "The IASLC lung cancer staging project: proposals for the inclusion of bronchopulmonary carcinoid tumors in the forthcoming (seventh) edition of the TNM classification for lung cancer," Journal of Thoracic Oncology, vol. 3, no. 11, pp. 1213-1223, 2008.

[16] N. Barouchos, A. Papazafiropoulou, N. Iacovidou et al., "Comparison of tumor markers and inflammatory biomarkers in chronic obstructive pulmonary disease (COPD) exacerbations," Scandinavian Journal of Clinical and Laboratory Investigation, vol. 75, no. 2, pp. 126-132, 2014.

[17] W. B. Wang, Y. G. Cui, and S. Y. Yao, "Message RNA expression of LUNX, CK19 and CEA genes in NSCLC with micrometastasis in lymph nodes," Chinese Journal of Oncology, vol. 30, no. 2, pp. 121-124, 2008.

[18] M. Tomita, T. Ayabe, E. Chosa, and K. Nakamura, "Correlation between serum carcinoembryonic antigen level and histologic subtype in resected lung adenocarcinoma," Asian Pacific Journal of Cancer Prevention, vol. 16, no. 9, pp. 3857-3860, 2015.

[19] M. Yamazaki, H. Ishikawa, R. Kunii et al., "Relationship between CT features and high preoperative serum carcinoembryonic antigen levels in early-stage lung adenocarcinoma," Clinical Radiology, vol. 69, no. 6, pp. 559-566, 2014.

[20] A. L. Picardo, A. J. Torres, M. Maestro et al., "Quantitative analysis of carcinoembryonic antigen, squamous cell carcinoma antigen, CA 125, and CA 50 cytosolic content in nonsmall cell lung cancer," Cancer, vol. 73, no. 9, pp. 2305-2311, 1994.

[21] J. J. Kim, K. Hyun, J. K. Park, and S. W. Moon, “The significance of serum carcinoembryonic antigen in lung adenocarcinoma," The Korean Journal of Thoracic and Cardiovascular Surgery, vol. 48, no. 5, pp. 335-344, 2015.

[22] S. Niho and T. Shinkai, "Tumor markers in lung cancer," Gan to Kagaku Ryoho Cancer \& Chemotherapy, vol. 28, no. 13, pp. 2089-2093, 2001.

[23] J. Y. Shin, S. J. Yoo, B. M. Park, S. S. Jung, J. O. Kim, and J. E. Lee, "Extremely increased serum carbohydrate antigen 19-9 levels caused by new or resistant infections to previous antibiotics in chronic lung diseases," Tuberculosis and Respiratory Diseases, vol. 75, no. 3, pp. 125-127, 2013.

[24] T. Goto, M. Hada, and T. Oyama, "Lung adenocarcinoma with clear cell features producing carbohydrate antigen 199," Asian Cardiovascular and Thoracic Annals, vol. 23, no. 8, pp. 985-987, 2015.

[25] H. Nagami, T. Nohara, M. Yamauchi, K. Yamada, and A. Nakase, "A case of two patients with primary lung cancer secreting CA19-9," Nihon Geka Gakkai Zasshi, vol. 89, no. 2, pp. 292-296, 1988.

[26] Y. Takano, T. Iwakiri, and Y. Ichiyasu, "A case of lung cancer producing CA19-9 and amylase," Nihon Kyōbu Shikkan Gakkai Zasshi, vol. 31, no. 2, pp. 267-270, 1993.

[27] J. L. Pujol, J. Grenier, J. P. Daures, A. Daver, H. Pujol, and F. B. Michel, "Serum fragment of cytokeratin subunit 19 measured 
by CYFRA 21-1 immunoradiometric assay as a marker of lung cancer," Cancer Research, vol. 53, no. 1, pp. 61-66, 1993.

[28] M. Hoefliger, R. Morant, W. Riesen, G. Baumgartner, and H. Engler, "CYFRA 21-1: initial experiences in bronchus carcinoma and other tumors," Schweizerische Medizinische Wochenschrift, vol. 124, no. 44, pp. 1966-1970, 1994.

[29] S. E. Kabawat, R. C. Bast, W. R. Welch, R. C. Knapp, and R. B. Colvin, "Immunopathologic characterization of a monoclonal antibody that recognizes common surface antigens of human ovarian tumors of serous, endometrioid, and clear cell types," American Journal of Clinical Pathology, vol. 79, no. 1, pp. 98-104, 1983.

[30] M. J. Gaspar, M. Diez, A. Rodriguez et al., "Clinical value of CEA and CA125 regarding relapse and metastasis in resectable non-small cell lung cancer," Anticancer Research, vol. 23, no. 4, pp. 3427-3432, 2003.

[31] G. Crombach, A. Scharl, M. Vierbuchen, H. Wurz, and A. Bolte, "Detection of squamous cell carcinoma antigen in normal squamous epithelia and in squamous cell carcinomas of the uterine cervix," Cancer, vol. 63, no. 7, pp. 1337-1342, 1989.

[32] K. Kagohashi, H. Satoh, H. Ishikawa, M. Ohtsuka, and K. Sekizawa, "A re-evaluation of squamous cell carcinoma antigen (SCC) as a serum marker for non-small cell lung cancer," Medical Oncology, vol. 25, no. 2, pp. 187-189, 2008.

[33] K. Kagohashi, H. Satoh, K. Kurishima et al., "Squamous cell carcinoma antigen in lung cancer and nonmalignant respiratory diseases," Lung, vol. 186, no. 5, pp. 323-326, 2008.

[34] M. Kotzsch, T. Kirchner, S. Soelch et al., "Inverse association of rab31 and mucin-1 (CA15-3) antigen levels in estrogen receptor-positive $(\mathrm{ER}+)$ breast cancer tissues with clinicopathological parameters and patients' prognosis," American Journal of Cancer Research, vol. 7, no. 9, pp. 1959-1970, 2017.

[35] D. Situ, J. Wang, Y. Ma et al., "Expression and prognostic relevance of MUC1 in stage IB non-small cell lung cancer," Medical Oncology, vol. 28, Supplement 1, pp. 596-604, 2011.

[36] X. Q. Liu, H. X. Ren, and Z. P. Wu, "Detecting MUC-1 mRNA for diagnosing peripheral blood micro-metastasis in non-small cell lung cancer patients," Chinese Journal of Cancer, vol. 27, no. 12, pp. 1267-1270, 2008. 


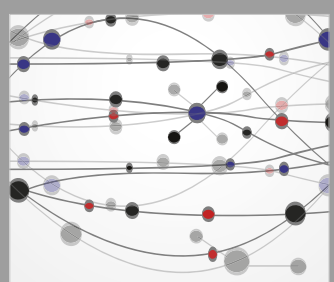

The Scientific World Journal
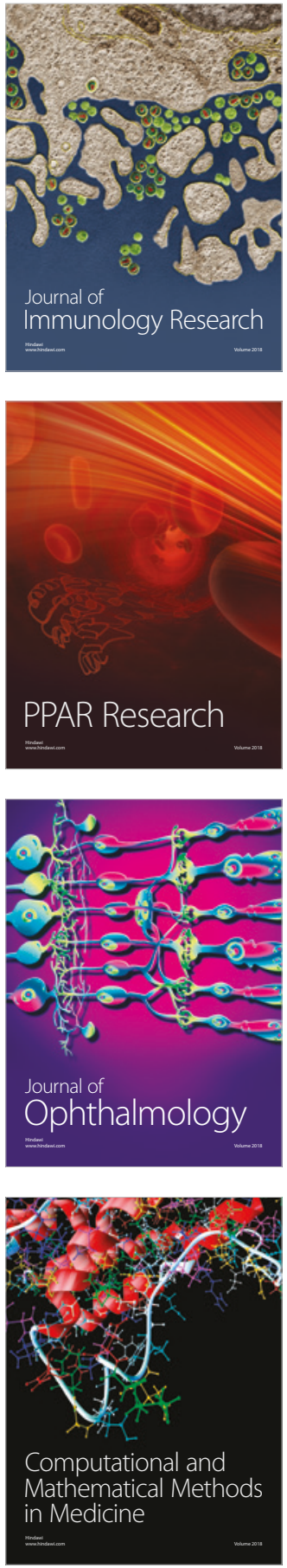

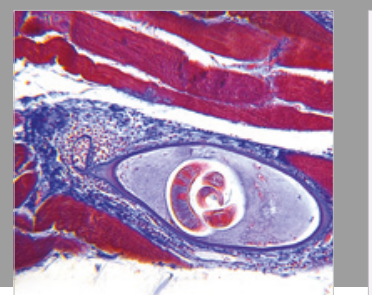

Gastroenterology Research and Practice

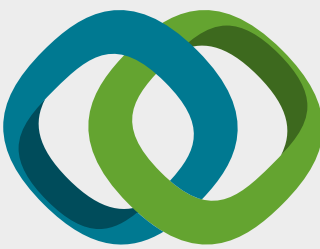

\section{Hindawi}

Submit your manuscripts at

www.hindawi.com
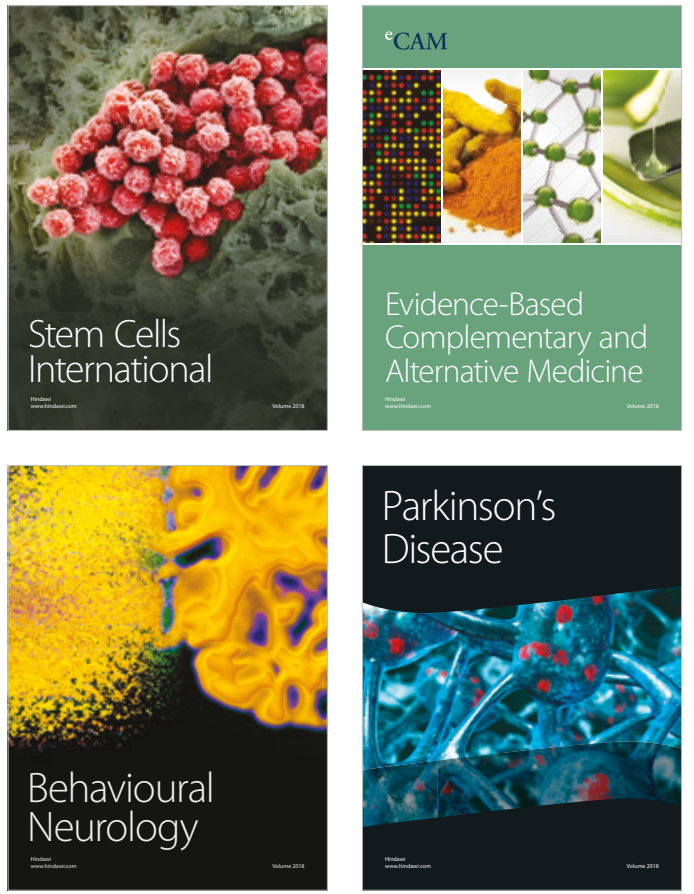

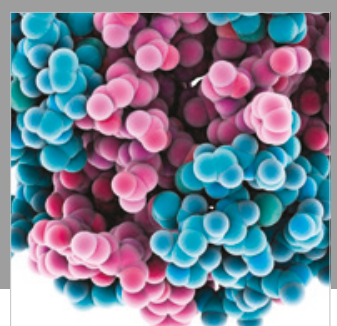

ournal of

Diabetes Research

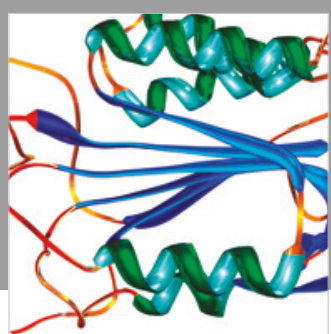

Disease Markers
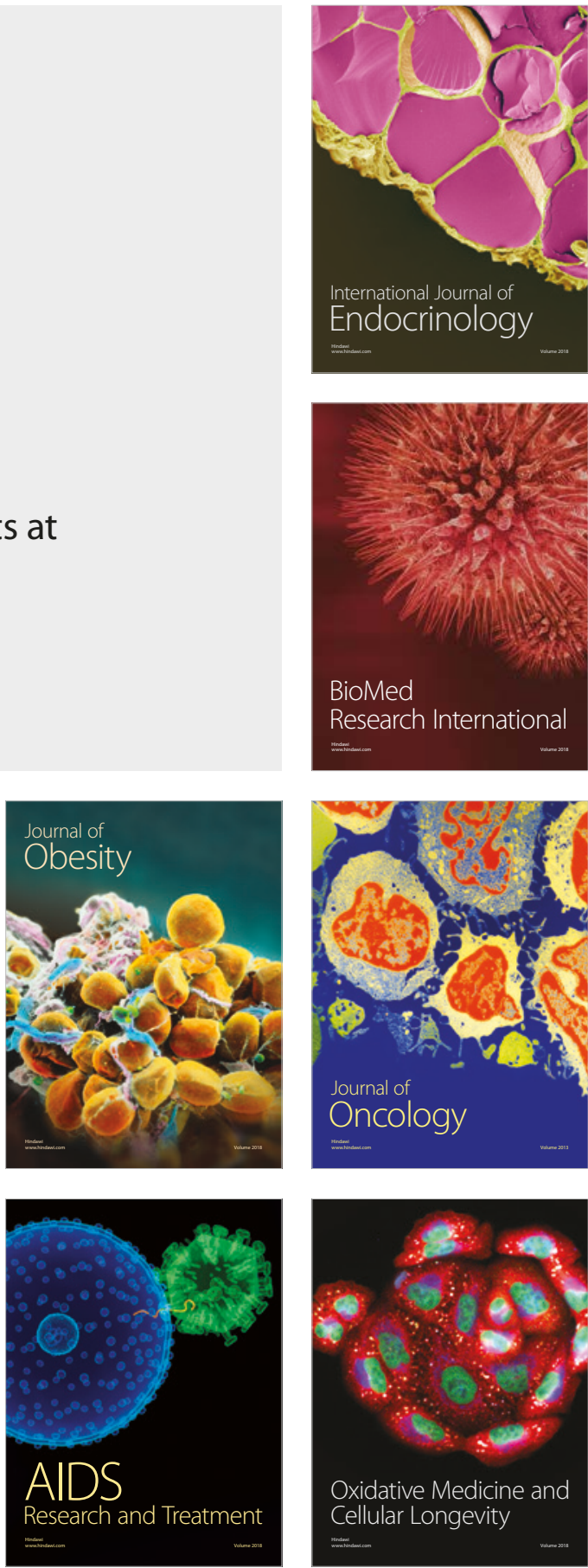